\title{
Is Contrast Enhanced Ultrasound (CEUS) ready for use in daily practice for evaluation of Focal Liver Lesions?
}

\section{Ioan Sporea, Roxana Șirli}

Department of Gastroenterology and Hepatology, "Victor Babeș" University of Medicine and Pharmacy Timișoara, Romania

\section{Abstract}

Abdominal ultrasound is one of the most popular imaging methods due to its feasibility, low cost and accessibility. Contrast Enhanced Ultrasound (CEUS) with second generation contrast agents became in the last years a useful tool for the characterization of focal liver lesions (FLL) so that EFSUMB issued guidelines for its use in clinical practice. Several large studies proved that CEUS has similar performance to more expensive imaging methods such as contrast enhanced CT and contrast enhanced MRI for the characterization of FLL. Also, several studies proved that CEUS is cost-effective as a first-line imaging method. Considering all these data, we think that CEUS is ready to be used in daily practice for the evaluation of FLL.

Keywords: contrast enhanced ultrasound, focal liver lesions, clinical practice, cost-effective

Abdominal ultrasound became the most popular imaging method due to its feasibility, low cost and accessibility. Ultrasound machines are better every day, a lot of image improvements becoming available in most devices (color Doppler, Power Doppler, or THI). Regarding the individuals who should perform ultrasound, they can be either radiologists (practice more common in Anglo-Saxon countries), or clinicians (gastroenterologists, urologists, internal medicine specialists, etc). Clinical ultrasound or "point of care" ultrasound [1] is a common practice for a long time in a part of Europe (Germany, Italy or Romania) and was recently "rediscovered" in the United States [2].

Since such a huge number of abdominal ultrasound examinations are performed in daily practice (either by

Received 15.12.2013 Accepted 14.01.2014

Med Ultrason

2014, Vol. 16, No 1, 37-40

Corresponding author: Roxana Şirli

Department of Gastroenterology and Hepatology,

"Victor Babeș" University of Medicine and

Pharmacy

10, Iosif Bulbuca Bv,

300736 Timisoara, Romania

Phone/Fax: + 40256488003

E-mail: roxanasirli@gmail.com clinicians or by radiologists), during which the liver structure should always be evaluated, a large number of focal liver lesions (FLL) are discovered. Some of them are the so called "incidentalomas" (incidental discovered lesions in asymptomatic subjects); others are lesions in patients known with chronic diseases (liver cirrhosis or oncologic patients). But, even if standard (grey scale) ultrasound is a sensitive method for the visualization of such lesions, it has low accuracy for their characterization.

In the last period, the use of Contrast Enhanced Ultrasound (CEUS) with second generation contrast agents ("bubbles") became a useful tool for the characterization of FLL. The evaluation time with CEUS is 4-5 minutes, and the conclusion can be drawn immediately after the examination. In 2004 the European Federation of Societies for Ultrasound in Medicine and Biology (EFSUMB) issued the first Guidelines concerning the use of contrast agents for the evaluation of FLL [3], which were updated in 2008 [4] and in 2012 [5]. The latest EFSUMB Guidelines [5], reflect a common point of view with the World Federation of Ultrasound in Medicine and Biology (WFUMB) (with the exception of USA, where second generation contrast agents are not approved by FDA).

Considering that CEUS has been proven to be an accurate method for FLL evaluation [and in the same time 
cheaper as compared with contrast enhanced spiral-CT (CE-CT) or contrast enhanced MRI (CE-MRI)], the question that arises is if the CEUS is ready to be used in daily practice for the evaluation of FLL.

Our answer is most certainly yes, and as an argument we will present a few large studies that proved the usefulness of CEUS for FLL characterization. The first two large national multicentre studies originated from Germany (DEGUM study) and France (STIC study), each one including more than 1000 lesions.

The German study [6] included 1,349 patients with FLL that could not be characterized by standard US alone. For each FLL analyzed, a diagnostic "gold standard" was available: biopsy in more than $75 \%$ of the lesions, CE-CT or CE-MRI in the rest of the cases. There were 573 benign lesions, and 755 malignant tumors; $62.3 \%$ of them incidentally discovered lesions, $17.3 \%$ discovered during the follow-up of patients with known liver cirrhosis and $27.0 \%$ in the follow-up of patients with known extrahepatic tumor. The overall accuracy of CEUS for the diagnosis of FLL was $90.3 \%$. CEUS had $95.8 \%$ sensitivity and $83.1 \%$ specificity, with $95.4 \%$ positive predictive value (PPV) and $95.9 \%$ predictive value (NPV) for differentiating benign vs. malignant lesions. Regarding the ability of CEUS to characterize different types of lesions, CEUS correctly diagnosed $82.2 \%$ of the hemangiomas, $87.1 \%$ of the focal nodular hyperplasias (FNHs), $57.9 \%$ of the adenomas, $84.9 \%$ of the hepatocellular carcinomas (HCCs) and $91.4 \%$ of the metastases.

The French multicentre study (STIC) [7] included 874 patients with 1034 FLL. In this study also, CEUS was compared to a reference method: CE-CT, CE-MRI or liver biopsy. Standard US correctly diagnosed $62.4 \%$ of the cases, while CEUS increased the diagnostic performance to $86.1 \%$. The diagnostic concordance between CEUS and the reference method was $73 \%$ (kappa $=0.67)$, better for FLL on non-cirrhotic liver ( $73.5 \%$, kappa $=0.66$ ), than in nodules on cirrhotic liver $(71.8 \%$, kappa $=0.42)$. CEUS had $79 \%$ sensitivity and $88 \%$ specificity for differentiating between benign vs. malignant FLLs. For the diagnosis of the most frequent FLL (hemangioma, FNH, metastases and HCC), the sensitivities were $85.4 \%, 82.5 \%$, $79.3 \%$ and $69.8 \%$ respectively, and the specificities were $93.7 \%, 94.3 \%, 92.5 \%$ and $94.7 \%$ respectively.

Sub-analyses were performed starting from the DEGUM study, considering some important and interesting issues. In such a study [8], the accuracy of CEUS was compared to CE-CT considering liver biopsy as the reference method. The assessment of tumor differentiation with CEUS and CE-CT was concordant in 124/158 cases and discordant in 30 cases (CEUS/CE-CT: sensitivity 94.0/90.7\%, specificity 83.0/81.5\%, PPV 91.6/91.5\%,
NPV 87.5/80.0\%, accuracy 90.3/87.8\%). A statistically significant difference regarding the accuracy of CEUS vs. CE-CT could not be established. If specific tumors such as hemangioma, FNH, HCC and metastases were analyzed, a statistically non significant slight advantage in tumor differentiation was found for CEUS.

In another sub-analysis [9] on a subgroup of patients from the DEGUM multicentre study, CEUS was compared to contrast MRI in 262 patients, in which the reference method was considered to be either CE-MRI for typical hemangioma and $\mathrm{FNH}$, or clinical evidence and additional follow-up (180 patients) or histology (82 patients). The subgroup in which biopsy was performed included mainly malignant lesions (55 cases), with 8 hemangiomas and 5 FNHs. Tumor differentiation was concordant in $56(68.3 \%)$ cases and tumor entity in 44 cases $(53.7 \%)$. Again there were no statistically significant differences between CEUS and MRI.

An advantage of CEUS is the possibility to dynamically evaluate the vascular pattern during the arterial, venous and late phases, thus allowing an accurate diagnosis in typical FLLs. Another study derived from the DEGUM multicentre study assessed the value of specific vascularization pattern in CEUS [10]. The patterns evaluated were: a wheel-spoke filling and arterial hyperenhancement followed by isoenhancement in the late phase in FNH; a nodular peripheral enhancement and partial or complete fill-in pattern in hemangiomas; portal and late phase hypoenhancement in metastases. Considering these typical vascular patterns, the diagnostic accuracy of CEUS was $83.1 \%$ for all benign lesions, $95.8 \%$ for all malignant ones, $91.4 \%$ for liver metastases and $84.9 \%$ for hepatocellular carcinomas.

In Romania we have one of the largest experiences regarding CEUS (data in press). In a prospective study performed in 14 centers from Romania, between February 2011 and June 2012, patients diagnosed with one to three de novo FLLs on B-mode ultrasound were evaluated with CEUS. In all cases, a reference method was available: CE-CT, CE-MRI or biopsy. FLLs were characterized during CEUS according to the EFSUMB Guidelines. For statistical analysis, indeterminate FLLs at CEUS were rated as false classifications. A total number of 536 cases were included in the final analysis, 344 malignant $(64.2 \%)$ and 192 benign lesions (35.8\%). The reference method was: CE-CT/MRI - 379 cases $(70.7 \%)$, pathological exam -150 cases $(27.9 \%)$ and aspiration of liver abscesses -7 cases $(1.4 \%)$. CEUS was conclusive in $89.3 \%$ cases and inconclusive in $10.7 \%$. To differentiate between malignant and benign FLLs, CEUS had 85.7 $\%$ sensitivity, $85.9 \%$ specificity, $91.6 \%$ PPV, $77.1 \%$ NPV and $85.8 \%$ accuracy. In this study, CEUS accuracy 
for differentiation between malignant and benign liver lesions was similar in tumors smaller than $2 \mathrm{~cm}$ vs. those with the diameter larger than $2 \mathrm{~cm}$. The conclusion of this study was that, in clinical practice, CEUS is a useful method for differentiating between malignant and benign FLLs, in concordance with the previous multicenter studies: DEGUM (Germany) and STIC (France) [6,7].

All the results of these multicenter, national studies are in favor of the use of CEUS in daily practice [6,7]. On the other hand, a large monocentric experience in which CEUS was performed by a group of experienced specialists in the field, showed similar results [11]. This retrospective study was performed by our group in the Gastroenterology and Hepatology Department of Timișoara and included 1254 FLLs (evaluated between September 2009 and October 2012). A CEUS examination was considered conclusive if the FLL respected the typical enhancement pattern as described in the EFSUMB Guidelines [4,5]. In this study, from the 1254 FLLs, CEUS was conclusive for a specific pathology in 1030 cases (82.1\%). Also, CEUS was able to differentiate benign vs. malignant lesions in $1111(90.6 \%)$ cases. Considering different categories of patients, the proportion of conclusive CEUS examinations was significantly higher in patients without chronic liver disease as compared with those with chronic hepatopathies: $86.4 \%$ vs. $73.8 \%, \mathrm{p}<0.0001$ [11]. Once again the conclusions of this study strongly recommend CEUS as a first line imaging method to characterize FLLs, at least in centers with a good experience in this domain.

As the final argument in favor of the use of CEUS for the evaluation of FLL we propose a recently published meta-analysis [12], for which literature databases were searched up to March 2012. Inclusion criteria were evaluation of CEUS in FLLs, assessment of sensitivity and specificity of CEUS for the diagnosis of malignant liver lesions. In this meta-analysis, the overall sensitivity and specificity of CEUS for the diagnosis of malignant liver lesions were $93 \%$ and $90 \%$ respectively. In a sub-analysis from this cohort of patients, no significant differences of specificity $(88 \%$ vs. $83 \%, p=0.11)$ was found between CEUS and CE-CT/MRI for the diagnosis of malignant liver lesions and the sensitivity of CEUS was significantly better than of CE-CT/MRI ( $95 \%$ vs. $89 \%, p=0.033$ ). Considering the characterization of specific FLLs, the overall sensitivity for the diagnosis of hemangioma (612 hemangiomas from 20 studies) was $86 \%$ and for the diagnosis of FNH (365 FNHs from 18 studies) was $88 \%$. The sensitivity for the diagnosis of HCC (1333 HCCs from 19 studies) was $88 \%$ and for the diagnosis of metastases (828 metastases from 18 studies) was 91\% [12]. The conclusion of this meta-analysis is to support the in- ternational recommendations on CEUS for the diagnostic work-up of focal liver lesions.

After the presentation of scientific data that certify the value of CEUS as a first line imaging method for the assessment of de novo FLLs, some data should be presented regarding the cost-effectiveness of this approach. Several studies were published in this regard.

In a sub-analysis of the French multicentre study [13], the diagnosis costs of 149 nodules were evaluated. The total savings were 128.5 Euros/nodule (considering as the mean cost of CEUS 155.2 Euros; of CE-CT 191.65 Euros; and of CE- MRI 322.3 Euros). In a German study [14] a cost-minimization analysis of CEUS as compared to CE-CT as the diagnostic standard for diagnosing incidental FLLs was conducted. The conclusion was that CEUS is the more cost-effective method in all scenarios in which CEUS were performed at specialized centers (122.18-186.53 Euros) as compared to CE-CT (223.19 Euros). In an Italian multicentre study [15] that included 485 subjects with 575 lesions, the total savings were 78,902 Euros, or 162 Euros/patient, if the classic patient work-up (baseline US followed by CE-CT or CE-MRI) was replaced with a new strategy in which baseline US was followed by CEUS. Data from a Romanian study also demonstrated that CEUS as the first line of diagnosis for FLL was the most cost-effective, with total savings of approximately 4,000 Euros as compared to CE-CT and approximately 24,900 Euros as compared to CE-MRI as first line imaging method for the characterization of 316 FLL [16].

Finally, a recently published systematic review [17] concluded that CEUS is a cost-effective replacement for CE-MRI. Also, CEUS was considered cost-effective as compared to CE-CT in the surveillance of cirrhosis and for the characterization of incidentally detected FLLs, with similar costs and effects for the detection of liver metastases secondary to colorectal carcinoma. Another important conclusion of this study was that CEUS had similar performance to CE-CT and CE-MRI for FLLs characterization.

Thus, considering the results of multicenter national experiences, of a large monocentric experience and of two meta-analyses (without forgetting the smaller price of CEUS as compared with other sectional imaging methods), we can conclude that in this moment CEUS is ready to be used in daily practice as a first line diagnostic method for incidentally discovered FLLs in standard abdominal ultrasound. In inconclusive cases, CE-CT/ MRI or echoguided biopsy should be used for the final diagnosis.

Conflict of interest: none 


\section{References}

1. Sporea I. Point of care or clinical ultrasound? Med Ultrason 2012; 14: 3-4.

2. Moore CL, Copel JA. Point-of-care ultrasonography. N Engl J Med 2011; 364: 749-757.

3. Albrecht T, Blomley M, Bolondi L, et al; EFSUMB Study Group. Guidelines for the use of contrast agents in ultrasound. January 2004. Ultraschall Med 2004; 25: 249-256.

4. Claudon M, Cosgrove D, Albrecht T, et al. Guidelines and good clinical practice recommendations for contrast enhanced ultrasound (CEUS) - update 2008. Ultraschall Med 2008; 29: 28-44.

5. Claudon M, Dietrich CF, Choi BI, et al. Guidelines and good clinical practice recommendations for Contrast Enhanced Ultrasound (CEUS) in the liver - update 2012: A WFUMB-EFSUMB initiative in cooperation with representatives of AFSUMB, AIUM, ASUM, FLAUS and ICUS. Ultrasound Med Biol 2013; 39: 187-210.

6. Strobel D, Seitz K, Blank W, et al. Contrast-enhanced ultrasound for the characterization of focal liver lesions-diagnostic accuracy in clinical practice (DEGUM multicenter trial). Ultraschall Med 2008; 29: 499-505.

7. Tranquart F, Le Gouge A, Correas JM, Ladam Marcus V, Manzoni P, Vilgrain V. Role of contrast-enhanced ultrasound in the blinded assessment of focal lesions in comparison with MDCT and CEMRI: Results from a multicentre clinical trial. EJC Suppl 2008;6:9-15.

8. Seitz K, Strobel D, Bernatik T, et al. Contrast-Enhanced Ultrasound (CEUS) for the characterization of focal liver lesions - prospective comparison in clinical practice: CEUS vs. CT (DEGUM multicenter trial). Parts of this manuscript were presented at the Ultrasound Dreiländertreffen 2008, Davos. Ultraschall Med 2009; 30: 383-389.

9. Seitz K, Bernatik T, Strobel D, et al. Contrast-enhanced ultrasound (CEUS) for the characterization of focal liver lesions in clinical practice (DEGUM Multicenter Trial): CEUS vs. MRI - a prospective comparison in 269 patients. Ultraschall Med 2010; 31: 492-499.
10. Strobel D, Seitz K, Blank W, et al. Tumor-specific vascularization pattern of liver metastasis, hepatocellular carcinoma, hemangioma and focal nodular hyperplasia in the differential diagnosis of 1,349 liver lesions in contrastenhanced ultrasound (CEUS). Ultraschall Med 2009; 30: 376-382.

11. Sporea I, Martie A, Popescu A, et al. Contrast Enhanced Ultrasound as first line imaging method for the evaluation of Focal Liver Lesions - A large monocentric experience. Ultrasound Med Biol 2013;39 Suppl:S81.

12. Friedrich-Rust M, Klopffleisch T, Nierhoff J, et al. Contrast-Enhanced Ultrasound for the differentiation of benign and malignant focal liver lesions: a meta-analysis. Liver Int 2013; 33: 739-755.

13. Tranquart F, Correas JM, Ladam Marcus V, et al. Echographie de contraste temps reel dans la prise en charge diagnostique des lesions nodulaires hepatiques: evaluation des performances diagnostiques et de l'impact economique sur une etude multicentrique francaise. J Radiol 2009; 90: 109122.

14. Giesel FL, Delorme S, Sibbel R, Kauczor HU, Krix M. Contrast-enhanced ultrasound for the characterization of incidental liver lesions - an economical evaluation in comparison with multi-phase computed tomography. Ultraschall Med 2009; 30: 259-268.

15. Romanini L, Passamonti M, Aiani L, et al. Economic assessment of contrast-enhanced ultrasonography for evaluation of focal liver lesions: a multicentre Italian experience. Eur Radiol 2007; 17 Suppl 6: F99-106.

16. Sirli R, Sporea I, Martie A, Popescu A, Dănilă M. Contrast enhanced ultrasound in focal liver lesions - a cost efficiency study. Med Ultrason 2010; 12: 280-285.

17. Westwood M, Joore M, Grutters J, et al. Contrast-enhanced ultrasound using SonoVue ${ }^{\circledR}$ (sulphur hexafluoride microbubbles) compared with contrast-enhanced computed tomography and contrast-enhanced magnetic resonance imaging for the characterisation of focal liver lesions and detection of liver metastases: a systematic review and cost-effectiveness analysis. Health Technol Assess 2013; 17: 241-243. 